\title{
TEST SPACES FOR NORMAL SPACES
}

\section{BYRON H. MCCANDLESS}

Let $Q$ be a class of topological spaces. For $X$ in $Q$, let $\operatorname{dim} X$ denote the dimension of $X$ defined in terms of finite open coverings. If $Q$ is the class of all normal spaces, then the dimension of spaces $X$ in $Q$ is characterized by the following theorem [2, p. 212]: $\operatorname{dim} X \leqq n$ if, and only if, given any closed subset $C$ of $X$, any continuous mapping of $C$ into the $n$-sphere $S^{n}$ has a continuous extension over $X$ with respect to $S^{n}$. The possibility of replacing $S^{n}$ in the above theorem by a more general space $Y$ was explored in two earlier papers $[8 ; 9]$. Such a space $Y$ we call a test space for $Q$-spaces of dimension $n$. To be precise, $Y$ is a test space for $Q$-spaces of dimension $n$ if it satisfies the statement: A $Q$-space $X$ has dimension $\leqq n$ if, and only if, given any closed subset $C$ of $X$, any continuous mapping of $C$ into $Y$ has a continuous extension over $X$ with respect to $Y$.

A space $Y$ is called an absolute neighborhood retract relative to the class $Q$ (abbreviated $\operatorname{ANR}(Q)$ ) if (a) $Y$ is in $Q$, and (b) whenever $Y$ is imbedded topologically as a closed subset of a $Q$-space $Z$, then $Y$ is a retract of some neighborhood of $Y$ in $Z$ (see [3]). Kodama has shown in [5] that an $n$-dimensional metric space $Y$ is an ANR (metric) if and only if $Y$ is $\mathrm{LC}^{n}$.

The results of $[8 ; 9]$ may be formulated as follows: if $Q$ is the class of all metric spaces, then an $n$-dimensional, $n$-LC, metric space $Y$ is a test space for $Q$-spaces of dimension $n$ if, and only if, $Y$ is an ANR(metric) and is the homotopy type of a wedge of $n$-spheres (i.e., a union of $n$-spheres with a single common point). In this paper we shall prove a similar theorem for $Q$ the class of all normal spaces.

In [9] we showed that if $Y$ and $Z$ are both ANR(metric) and of the same homotopy type, then $Y$ is a test space for metric spaces of dimension $n$ if and only if $Z$ is. Needed in the proof was Borsuk's homotopy extension theorem. Dowker showed in [2, p. 205] that the homotopy extension theorem holds for mappings of a normal space into an ANR(compact metric) provided homotopy is replaced by uniform homotopy. Using this result, we can prove the following lemma:

Lemma 1. Let $Y$ and $Z$ be $A N R$ (compact metric) having the same homotopy type. Then $Y$ is a test space for normal spaces of dimension $n$ if and only if $Z$ is.

Received by the editors April 24, 1961. 
Proof. Let us use Kuratowski's notation $X \tau Y[7$, p. 251] to mean that if $C$ is a closed subset of $X$, then any continuous mapping of $C$ into $Y$ has a continuous extension over $X$.

Suppose that $Z$ is a test space for normal spaces of dimension $n$; we shall show that $Y$ is also. We first show that $Y$ satisfies the condition: $X \tau Y$ implies $\operatorname{dim} X \leqq n$ for any normal space $X$. To do this, let $X$ be any normal space satisfying $X \tau Y, C$ any closed subset of $X$, and $f: C \rightarrow Y$ any continuous mapping. Since $Y$ and $Z$ have the same homotopy type, there exist continuous mappings $\alpha: Y \rightarrow Z$ and $\beta: Z \rightarrow Y$ such that $\alpha \beta$ and $\beta \alpha$ are homotopic to the identity mappings of $Z$ and $Y$ respectively. Since $Y$ and $Z$ are compact metric, it follows from another result of Dowker [2, p. 227] that $\alpha \beta$ and $\beta \alpha$ are $u n i$ formly homotopic to these identity mappings. Consider the composition mapping $\beta f: C \rightarrow Y$. Since we are given $X \tau Y, \beta f$ has a continuous extension $F: X \rightarrow Y$, and this provides a mapping $\alpha F: X \rightarrow Z$. Now $\alpha F \mid C=\alpha \beta f$, and since $\alpha \beta$ is uniformly homotopic to the identity mapping of $Z$, it follows that $\alpha \beta f$ is uniformly homotopic to $f$. Dowker's version of Borsuk's homotopy extension theorem now yields a continuous extension of $f$ over $X$. Therefore $\operatorname{dim} X \leqq n$, and the first part of the theorem is proved.

To complete the proof that $Y$ is a test space for normal spaces of dimension $n$, it remains to be shown that $\operatorname{dim} X \leqq n$ implies $X \tau Y$ for any normal space $X$. This proof is quite similar to the one above, and will not be given.

As in $[10$, p. 209] we denote by $X \vee Y$ the "wedge product" of the spaces $X$ and $Y$, that is, the one-point union of $X$ and $Y$. If $f: X \rightarrow Z$ and $g: Y \rightarrow Z$ are mappings which agree on the common point, then a mapping $f \vee g: X \bigvee Y \rightarrow Z$ is defined by letting $f \vee g$ be equal to $f$ on $X$ and equal to $g$ on $Y$. The wedge product will be useful in the following lemma and theorems.

Lemma 2. A finite wedge of $n$-spheres is a test space for normal spaces of dimension $n$.

Proof. This follows as in $[8 ; 9]$ since the $n$-sphere tests the dimension of arbitrary normal spaces [2, p. 212].

Lемма 3. If the compact metric space $Y$ is a test space for compact metric spaces of dimension $n$, then

$$
\pi_{i}(Y)=0 \text { for } i<n, \text { and } Y \text { is } \mathrm{LC}^{n-1} .
$$

Proof. This is clearly a corollary to a theorem of Kuratowski $[7$, p. 266] who proved that the conclusion is equivalent to the prop- 
erty: $\operatorname{dim} X \leqq n$ implies $X \tau Y$ for a separable metric space $Y$. A test space for compact metric spaces, of course, has this property.

We can now prove the main results of this paper.

Theorem 1. Let $Y$ be a compact, $n$-LC, $n$-dimensional metric space. If $Y$ is a test space for normal spaces of dimension $n$, then $Y$ is an $A N R$ (metric) and has the homotopy type of a finite wedge of $n$-spheres

Proof. The case $n=1$ requires separate treatment because of the non-abelian character of $\pi_{1}(Y)$. By hypothesis, $Y$ is a compact 1dimensional, 1-LC metric space, so by Lemma 3 and Kodama's result [5] $Y$ is an ANR(metric). It follows that $Y$ is locally connected and locally simply connected, so by a result of Curtis and Fort [1, p. 143] $\pi_{1}(Y)$ is finitely generated and free. Therefore $\pi_{1}(Y)$ is isomorphic to the fundamental group of finite wedge of 1-spheres. Let $f_{i}: S^{1} \rightarrow Y$ be a finite set of base-point preserving mappings which generate $\pi_{1}(Y)$. Then it is clear that $\mathrm{V}_{i}: V^{1} \rightarrow Y$ induces an isomorphism of $\pi_{1}\left(V S^{1}\right)$ onto $\pi_{1}(Y)$. Now $V S^{1}$ and $Y$ are both dominated by 1-dimensional CW complexes [4, p. 365] so the "classical theorem" of Whitehead [11, Theorem 3, p. 216] shows that $V f_{i}$ is a homotopy equivalence. Therefore $V S^{1}$ and $Y$ have the same homotopy type and this completes the proof in the case $n=1$.

Suppose now that $n \geqq 2$. Then the hypotheses imply that $Y$ is an $n$-dimensional, $\mathrm{LC}^{n}$ metric space, so by Kodama's theorem, $Y$ is an ANR(metric). Thus [6] the singular and Cech homology groups are isomorphic in each dimension, and since $Y$ is compact, they are finitely generated. Since $Y$ is $n$-dimensional, $H_{i}(Y)=0$ for $i>n$ and $H_{n}(Y)$ is torsion-free. By Lemma 3 and the Hurewicz Isomorphism Theorem we have $H_{n}(Y) \approx \pi_{n}(Y)$ and $H_{i}(Y)=0$ for $i<n$; hence $H_{n}(Y)$ is the only nonzero homology group of $Y$. Let $f_{i}: S^{n} \rightarrow Y$ be a set of base-point preserving mappings which generate $\pi_{n}(Y)$. Then $\mathrm{V} f_{i}: \mathrm{V} S^{n} \rightarrow Y$ induces an isomorphism of $\pi_{n}\left(\mathrm{~V} S^{n}\right)$ onto $\pi_{n}(Y)$ and therefore of $H_{n}\left(\mathrm{~V} S^{n}\right)$ on $H_{n}(Y)$. It now follows from the Whitehead theorem that $\mathrm{V}_{i}$ is a homotopy equivalence. Therefore $Y$ has the homotopy type of $V S^{n}$ and this completes the proof of the theorem.

Theorem 2. If $Y$ is an ANR(compact metric) and has the homotopy type of a finite wedge of $n$-spheres, then $Y$ is a test space for normal spaces of dimension $n$.

Proof. By Lemma 2, a finite wedge of $n$-spheres is a test space for normal spaces of dimension $n$. Since a finite wedge of $n$-spheres is an ANR(compact metric), it follows from Lemma 1 that $Y$ is also a test space for normal spaces of dimension $n$. 
Note that if $Y$ is a test space for a certain class of spaces, it is also a test space for any subclass. Thus, a test space for normal spaces of dimension $n$ is also a test space for compact metric spaces of dimension $n$. It is likewise clear that a compact ANR(metric) is an ANR (compact metric). Therefore Theorems 1 and 2 imply

Theorem 3. Let $Y$ be a compact, $n$-dimensional, $n$-LC metric space. Then $Y$ is a test space for normal spaces of dimension $n$ if and only if it is an $A N R$ (compact metric) and has the homotopy type of a finite wedge of $n$-spheres.

In closing, the author would like to acknowledge some suggestions of the referee which led to a strengthening of the final result.

\section{BIBLIOGRAPHY}

1. M. L. Curtis and M. K. Fort, Jr., The fundamental group of one-dimensional spaces, Proc. Amer. Math. Soc. 10 (1959), 140-148.

2. C. H. Dowker, Mapping theorems for non-compact spaces, Amer. J. Math. 69 (1947), 200-241.

3. $O$. Hanner, Retraction and extension of mappings of metric and non-metric spaces, Ark. Mat. 2 (1954), 315-360.

4. J. Dugundji, An extension of Tietze's theorem, Pacific J. Math. 1 (1951), 353-367.

5. Y. Kodama, On LCn metric spaces, Proc. Japan Acad. 33 (1957), 79-83.

6. - On ANR for metric spaces, Sci. Rep. Tokyo Kyoiku Daigaku 5 (A) (1955), 96.

7. C. Kuratowski, Topologie. II, Warszawa-Wroclaw, 1950.

8. B. H. McCandless, Test spaces for dimension n, Proc. Amer. Math. Soc. 7 (1956), 1126-1130.

9. - Test spaces for metric spaces, Proc. Amer. Math. Soc. 10 (1959), 372376.

10. G. W. Whitehead, A generalization of the Hopf invariant, Ann. of Math. 51 (1950), 192-237.

11. J. H. C. Whitehead, Combinatorial homotopy. I, Bull. Amer. Math. Soc. 55 (1949), 213-245.

Rutgers, The State University 